
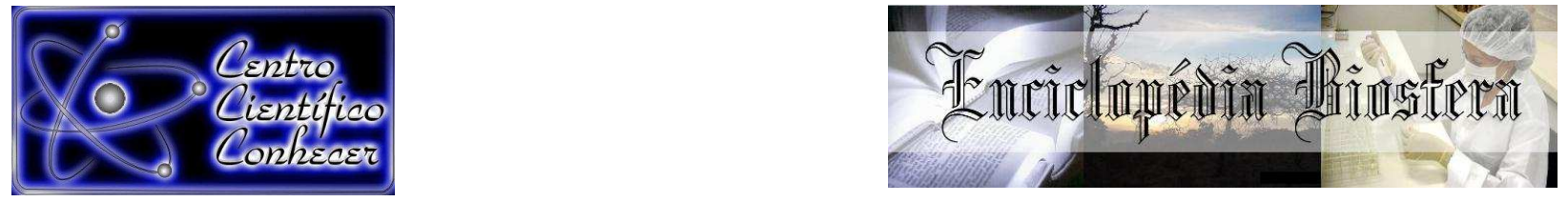

\title{
COMPORTAMENTO DE GENÓTIPOS DE PIMENTEIRA-DO-REINO CULTIVADOS EM DOIS TIPOS DE TUTORES
}

\author{
Simone de Miranda Rodrigues ${ }^{1}$, Marli Costa Poltronieri ${ }^{2}$, Oriel Filgueira de Lemos $^{2}$ \\ ${ }^{1}$ Pesquisador da Embrapa Amazônia Oriental, Belém - Pará, Brasil. Travessa Dr. \\ Enéas Pinheiro s/n. CEP 66095-100 (simone.rodrigues@embrapa.br) \\ ${ }^{2}$ Pesquisador da Embrapa Amazônia Oriental, Belém - Pará
}

Recebido em: 02/10/2017 - Aprovado em: 21/11/2017 - Publicado em: 05/12/2017 DOI: 10.18677/EnciBio_2017B17

\begin{abstract}
RESUMO
O cultivo da pimenta-do-reino em estacão tem se mostrado oneroso na sua implantação assim como desfavorável ao meio ambiente, implicando na necessidade da condução dos pimentais de modo mais sustentável. A gliricídia, como tutor vivo, apresenta baixo custo de implantação, podendo ser considerada excelente alternativa a agricultores familiares. Propôs-se avaliar o comportamento de cinco cultivares de pimenteira-do-reino em estacão e gliricídia no Pará, Brasil. Foram avaliadas 20 plantas de cada cultivar em cada tipo de tutor. Realizou-se o levantamento de plantas mortas e o rendimento de pimenta preta por cultivar,por ano, considerando o tipo de tutor. A cultivar Uthirankotta apresentou menor mortalidade no primeiro ano, enquanto as cultivares Cingapura e cv. Embrapa apresentaram maior porcentagem de mortalidade no segundo ano. O cultivo em gliricídia evidenciou que a Uthirankotta e cv. Embrapa foram as mais produtivas e melhor adaptadas ao cultivo semi-intensivo, enquanto que a Bragantina apresentou maior produtividade quando cultivada em tutor morto. Como o cultivo em estacão promove os melhores resultados de produção para as cultivares, justifica-se a necessidade de adequação dos sistemas nutricional e de irrigação para pimenteirado-reino cultivada em gliricídia.
\end{abstract}

PALAVRAS-CHAVE: Gliricidia sepium L. Piper nigrum L. Tutor morto.

\section{BEHAVIOR OF BLACK PEPPER GENOTYPES GROWN ON TWO TYPES OF STAKES}

\begin{abstract}
Black pepper cultivation on stake has been costly in its implantation as well as unfavorable to the environment, implying in the necessity of the management of the plants in a more sustainable way. Gliricidia, as a black pepper living stake, presents low cost of implantation and is being considered an excellent alternative to familiar farmers. It was proposed to evaluate the behavior of five black pepper cultivars using dead stake and Gliricidia in Pará, Brazil. It was evaluated 20 plants of each cultivar in each type of stake. It was carried out the survey of dead plants and the yield of black pepper per cultivar, per year, considering the type of tutor. Uthirankotta cultivar showed lower mortality in the first year, while the cultivar Cingapura and Embrapa had a higher percentage of mortality in the second year. The cultivation in Gliricidia
\end{abstract}


evidenced that Uthirankotta and cv. Embrapa were the most productive and most adapted to semi-intensive cultivation, while Bragantina presented higher productivity when grown using dead stake. As cultivation in dead stake promotes the best results of production for the cultivars, it is justified the need of adaptation of the irrigation and nutritional systems for black pepper cultivated in Gliricidia.

KEYWORDS: Dead stake. Gliricidia sepium L. Piper nigrum L.

\section{INTRODUÇÃO}

Originada da Índia, a pimenta-do-reino (Piper nigrum L.) é um dos principais produtos agrícolas na pauta de exportação da região Amazônica tornando o Brasil o quarto maior produtor do mundo, sobretudo na forma de pimenta preta, que constitue o principal produto de comercialização (PEREIRA et al., 2014; ANDRADE et al., 2017). Cerca de $85 \%$ da produção nacional provem da agricultura familiar, gerando 30 mil empregos diretos durante o ano, e chegando a 80 mil na época da colheita. Possui alto valor econômico, sendo amplamente utilizada na agroindústria, na indústria alimentícia e química (EGER, 2011; ASSIS et al., 2015).

O Brasil assumiu o cultivo comercial a partir da década de 30 no Pará, se tornando o maior exportador mundial na década de 80 (FILGUEIRAS et al., 2014). Atualmente, o estado detém mais de $66 \%$ da produção nacional, seguido do Espírito Santo e Bahia (IBGE, 2017). Como é uma planta trepadeira, requer o uso de tutor para o desenvolvimento, o quê permite obter considerável produção. Normalmente é cultivada de modo intensivo, a pleno sol, usando estacões obtidos de madeira de lei, ecologicamente inviável.

Ishizuka et al. (2004) afirmaram que a cada hectare cultivado de pimenteirado-reino é necessário derrubar de 25 a 30 árvores de madeira-de-lei para a produção de estacões. Visando minimizar custos, danos ocasionados ao meio ambiente e adequar a legislação vigente foi desenvolvido outro sistema de cultivo para a pimenteira-do-reino no Brasil, usando gliricídia (Gliricidia sepium L.), mas ainda pouco utilizado, apesar da redução dos custos de implantação dos pimentais usando esse tipo de tutor (KATO et al., 1997). Os custos para implantação são consideravelmente menores, já que uma estaca de gliricídia custa cerca de $R \$ 2,50$, enquanto o estacão custa em média $R \$ 15,00$, sem considerar as limitações com a obtenção desse tipo de tutor, visto a legislação ambiental que protege espécie produtoras de madeira de lei. Portanto, esse tipo de tutor poderá contribuir para aumentar a sustentabilidade econômica da pipericultura, devido não apenas à escassez de madeira de lei e redução do desmatamento da floresta, mas também, reduzir os altos custos para obtenção dos tutores.

Comparando-se o custo de implantação do pimental nos dois tipos de tutores, observa-se uma redução de $21 \%$ no primeiro ano de cultivo, devido os custos baixos para aquisição dos tutores vivos e um acréscimo de mão de obra, nos anos subsequentes, devido à necessidade de poda da gliricídia (MENEZES et al., 2016). Apesar da possibilidade do uso de gliricídia como tutor vivo da pimenteira-do-reino, há poucos dados a respeito do comportamento de cultivares de pimenteira-do-reino conduzidas com esse tutor. Em decorrência da escassez de informações, esse trabalho se propôs avaliar, quanto a aspectos produtivos, cinco cultivares de pimenteira-do-reino plantadas em tutores morto e vivo no estado do Pará. 


\section{MATERIAL E MÉTODOS}

O estudo foi realizado no município de Igarapé-Açú, Pará-Brasil, pertencente à Mesorregião do Nordeste Paraense e Microrregião Bragantina, durante os anos de 2012 a 2016. Os dados experimentais foram obtidos de uma fazenda localizada em

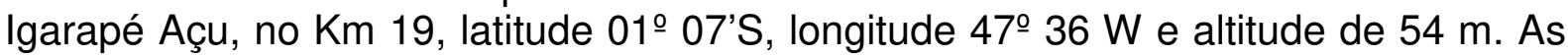
condições climáticas da região são do tipo Am de acordo com a classificação de Koppen. Sua média de precipitação é de $2495 \mathrm{~mm}$, com média de temperatura anual de $26.4 \stackrel{\circ}{\circ}$. Em janeiro de 2013, foi instalada uma Unidade Experimental de pimentado-reino que consistiu no plantio de cinco cultivares: Bragantina, Cingapura, Uthirankotta, Kuthiravally e cv. Embrapa (obtida via programa de melhoramento da Embrapa), as quais foram plantadas em tutor morto e tutor vivo para avaliação do comportamento dessas cultivares nesses dois tipos de tutores.

Foram utilizadas 20 mudas de cada cultivar dispostas em duas linhas contendo 10 plantas por linha para cada tipo de cultivar, totalizando 10 linhas para cada tipo de tutor. Quatro plantas a mais foram plantadas em cada fileira, sendo consideradas bordaduras, não sendo contabilizadas. O espaçamento utilizado foi de 2,25 por 2,25 m entre os tutores de fila dupla, e $4 \mathrm{~m}$ entre as filas duplas, totalizando $22,50 \mathrm{~m}$ o comprimento de cada fileira e $27,25 \mathrm{~m}$ o conjunto das cinco cultivares para cada tipo de tutor. As estacas de gliricídias de $2,5 \mathrm{~m}$ de comprimento e $5 \mathrm{~cm}$ de diâmetro foram plantadas em dezembro de 2012, sendo enterradas $50 \mathrm{~cm}$ no solo, mesma época da introdução dos estações. Em janeiro de 2013, as mudas das cultivares de pimenteira-do-reino foram plantadas a $10 \mathrm{~cm}$ dos tutores.

As adubações em todos os anos ocorreram no período chuvoso (janeirojunho), conforme recomendação usada para a pimenteira-do-reino (FRANZINI et al., 2014), consistindo de $130 \mathrm{~g} /$ planta de sulfato de amônio (21\% N), $120 \mathrm{~g} /$ planta de superfosfato simples $\left(18 \% \mathrm{P}_{2} \mathrm{O}_{5}\right)$ e $50 \mathrm{~g} /$ planta de cloreto de potássio $\left(21 \% \mathrm{~K}_{2} \mathrm{O}\right)$, os quais foram divididos em três aplicações. As inflorescências e espigas que se formaram ao final do primeiro ano de plantio foram retiradas, visando melhor desenvolvimento das plantas no ano seguinte, sendo considerado nas análises as colheitas do segundo e terceiro ano. Após os períodos de colheita, em dezembro, utilizou-se $20 \mathrm{mg} / \mathrm{L}$ do complexo 151 para adubação foliar e reestabelecimento das plantas.

As gliricídias foram conduzidas conforme recomendação de Menezes et al. (2013), e o rendimento das cultivares no segundo e terceiro ano foi verificado considerando a média de produção de semente seca por planta viva, sendo realizada a cata das espigas e secamento dos grãos em terreiro a pleno sol. Ao final de 2015 e 2016 foi contabilizado o número de plantas mortas de cada cultivar por tipo de tutor, visando o replantio dos materiais, sendo a mortalidade convertida para porcentagem. O monitoramento do comportamento das cultivares em tutores vivo e morto foi realizado registrando-se o crescimento das cultivares nos tutores por meio do levantamento da mortalidade das plantas e comparação entre as médias da produção por cultivar.

\section{RESULTADOS E DISCUSSÃO}

A diminuição da oferta de madeira como tutor tem elevado o preço da produção. No caso da pimenta-do-reino foi gerada uma tecnologia de plantio utilizando G. sepium L., já adotada por produtores, por ser possível seu plantio em forma de estaca, permitir sua poda e ser fixadora de nitrogênio (MENEZES, 2014). Entretanto, são escassos os estudos avaliando a produção de materiais genéticos 
de pimenteira-do-reino cultivados nessas condições. Nesse estudo, durante o período de avaliações ocorreram perdas de plantas no campo em decorrência do período prologado de estiagem e ocorrência da murcha amarela, doença causada pelo fungo Fusarium oxysporum, nas cultivares implantadas em 2014 em Igarapé Açu-PA, objeto deste estudo comparativo. Em 2015, as cultivares Embrapa e Bragantina foram as que apresentaram maiores índices de mortalidade quando cultivadas em tutor morto, cerca de $65 \%$ e $50 \%$ das plantas, respectivamente, enquanto a cultivar Uthirankotta apresentou a menor perda, em torno de $25 \%$ (Figura 1), confirmando as informações de Poltronieri e Lemos (2014) de que a cultivar Bragantina tem resistência média à murcha amarela enquanto as demais, com exceção da cultivar Embrapa, ainda sem avaliação para este parâmetro, têm alta tolerância.

As cultivares Cingapura e Kuthiravally em tutor vivo, gliricídia, apresentaram mortalidade na ordem de $65 \%$ e $55 \%$, respectivamente, enquanto em 2015 , as cultivares Embrapa e Cingapura apresentaram índice de mortalidade de 58\% e 55\%, respectivamente (Figura 1). A tolerância à estresse hídrico e às principais doenças na cultura, o uso de fertilizantes e à competição com outras plantas tem influência no comportamento de genótipos de pimenteira-do-reino em campo (RODRIGUES et al., 1987; VELOSO et al., 2000; ALBUQUERQUE; DUARTE, 1991).

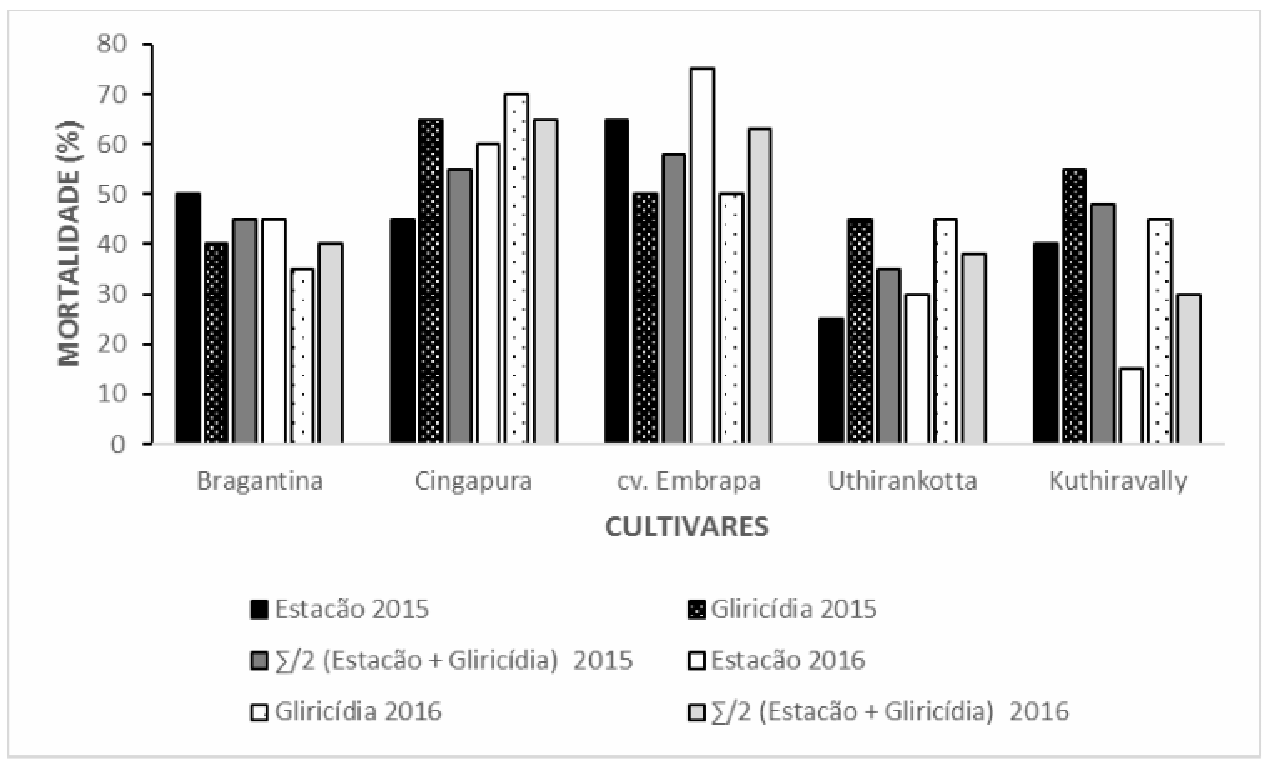

FIGURA 1 - Mortalidade de plantas de pimenteira-do-reino nos anos avaliados.

Em 2016, considerando as condições do solo arenoso e déficit hídrico no município, devido às condições climáticas proporcionadas pelo El Ninõ, as perdas de plantas continuaram após replantio, destacando a cv. Embrapa que apresentou índice elevado de mortalidade quando cultivada em estacão, chegando a uma perda de $75 \%$ das plantas, enquanto a cv. Cingapura apresentou índice de $70 \%$ de mortalidade em cultivo sombreado (gliricídia). As plantas das cultivares Uthirankotta e Kuthiravally sofreram perdas menores com índices inferiores a $50 \%$. Neste caso, a qualidade das mudas com sistema radicular abundante, bem desenvolvido é fundamental para suportar o período de estresse hídrico (FREIRE, 2013). Perdas de plantas em experimento semelhante resultou na perda de cerca de $30 \%$, como relatado por Conceição et al. (2003). 
Quanto à produção, tomada a partir de 2015, dentre as cultivares em tutor do tipo gliricídia, a cv. Uthirankotta se destacou naquele ano, apresentando $1,26 \mathrm{Kg}$ de pimenta preta, e considerando os dois tutores, $1,88 \mathrm{~kg}$ para a média de produção, enquanto em 2016, a média de produção total foi de $1,76 \mathrm{Kg}$ de pimenta preta (Figura 2). Conceição et al. (2003) observaram que a cultivar Guajarina se destacou quando cultivada em gliricídia, no espaçamento de 2,5 X 2,5 X 5,0m com média de $2.706,60 \mathrm{Kg} / \mathrm{ha}$ seguida da cultivar Cingapura com 1.889,00 Kg/ha. Esta última teve uma boa performance também neste trabalho com produção inferior à cv. Embrapa e Uthirankotta. Ressalte-se que a maioria das cultivares apresentaram maior produção em tutor morto, estacão, o que se deve à competição por nutrientes entre as duas plantas, considerando que a adubação não foi alterada para ambos os tutores no sistema de produção, ou seja, permaneceu a mesma. Quando cultivada em tutor morto, a cv. Uthirankotta mostrou-se bastante exigente em termos nutricionais no fim do período de colheita (novembro/dezembro), apesar de apresentar maior tolerância ao período seco, em decorrência de ser uma cultivar com produção tardia (novembro), período de menor precipitação pluviométrica no nordeste paraense (POLTRONIERI; LEMOS, 2014). A cv. Kuthiravally só apresentou produção em 2016, com média menor que $0,1 \mathrm{Kg}$ de pimenta por pé, o que diferiu do observado por Conceição et al. (2003).

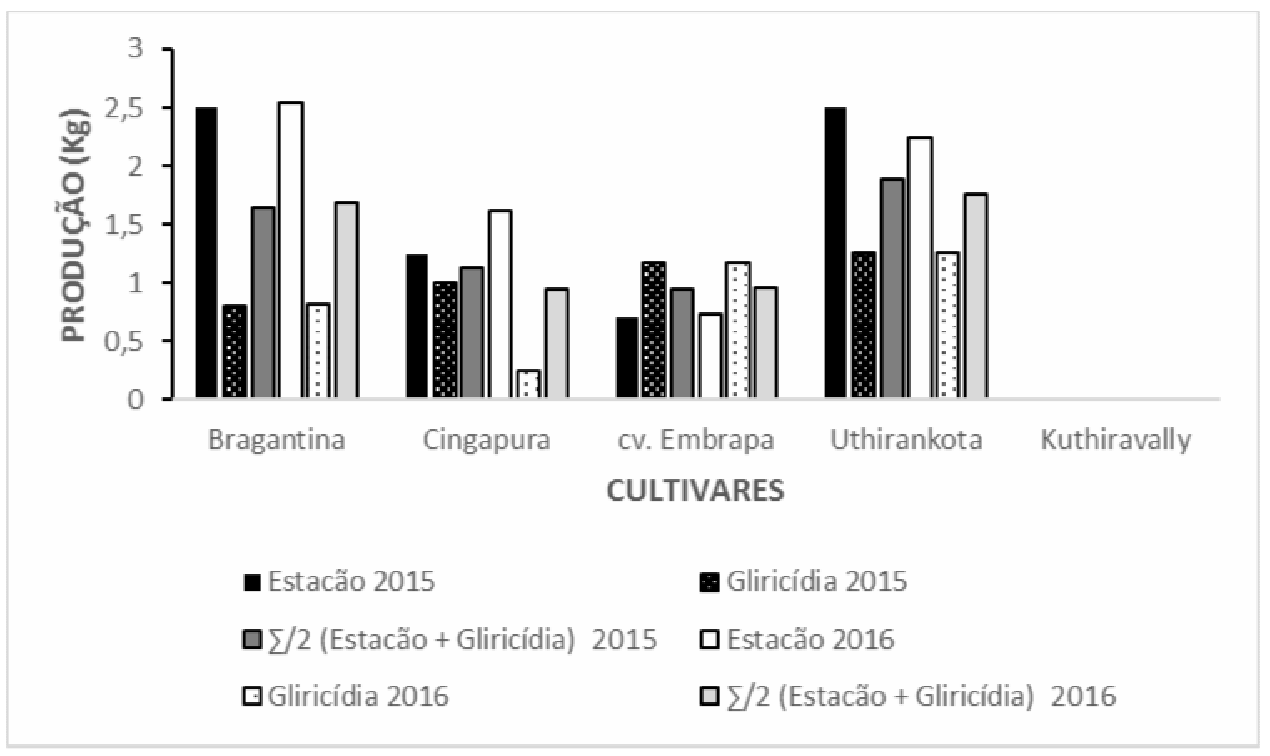

FIGURA 2 - Média da produção de pimenta-do-reino por planta.

O cultivo em gliricídia evidenciou uma estabilidade da produção nos anos avaliados, exceto para a cultivar Cingapura que apresentou brusca redução da produção média por planta ao fim do segundo ano de cultivo, sendo o tutor morto mais adequado para essa cultivar. Albuquerque e Duarte (1991) observaram comportamento diferente quanto a produção de pimenta preta em tutor morto, com destaque para a cultivar Guajarina com cerca de $2,5 \mathrm{Kg} /$ planta de pimenta preta, enquanto a cultivar Cingapura produziu $1,05 \mathrm{Kg} /$ planta.

$\mathrm{Na}$ condução das plantas em tutor vivo (gliricídia), as cultivares Uthirankotta e Embrapa apresentaram maior produção, enquanto a Bragantina mostrou produção mais baixa. Com relação às cultivares conduzidas em tutor morto, com exceção da cultivar Embrapa, todas as cultivares apresentaram produção satisfatória, entretanto, 
as cultivares Uthirankotta, Bragantina e Cingapura foram as que mais se destacaram. Em geral, dois materiais, Uthirankotta, Bragantina, tiveram maior produção nas condições de cultivo a pleno sol (tutor morto), apresentando um pequeno aumento na produção a partir do segundo ano.

Os fatores climáticos (deficiência hídrica no solo devido ausência de chuvas em decorrência ao prolongado período de estiagem no município) e bióticos (ocorrência do fungo $F$. oxysporum) foram determinantes nas perdas, morte das plantas nos anos avaliados, com também observado por Conceição et al. (2003) que obtiveram perdas devido a ocorrência da doença fusariose ( $F$. solani f. sp. piperis). De acordo com dados agrometeorológicos obtidos pelo Inmet (2017), o município de Igarapé Açu recebeu reduzida precipitação pluviométrica entre os meses de outubro a dezembro de 2015 e 2016, comparadas à média anual dos anos anteriores.

A avaliação de cultivares de pimenteira-do-reino em tutor vivo carece de pesquisas e informações. Segundo Menezes et al. (2013), o tutor vivo pode ser produzido na própria fazenda, podendo ser incorporado no solo, funcionando como cobertura morta, além de reduzir a temperatura e a ventilação excessiva no pimental. Conceição et al. (2003), comparando cinco cultivares, verificaram que a cv. Guajarina foi a mais adequada ao cultivo em gliricídia em Tomé Açu-PA, enquanto que a cv. Cingapura foi considerada a menos produtiva.

As cultivares de pimenteira-do-reino apresentam diferentes comportamentos à diversos fatores, em resposta à adubação orgânica com esterco bovino (OLIVEIRA et al., 2007), sintomas de deficiência nutricionais (VELOSO et al., 2000), resposta ao incremento de produção com adição de potássio para as cultivares Cingapura, Bragantina e Guajarina, e resposta à adição de fósforo somente para a cultivar Guajarina (VELOSO et al., 2000); além disso, todas as cultivares são suscetíveis à doença fusariose com grande incidência a partir do terceiro ano, sendo os pimentais totalmente dizimados após o sexto ano de cultivo (ALBUQUERQUE; DUARTE, 1991). Ademais, considerando a escassez de trabalhos com pimenta-do-reino em sistemas de tutor vivo, esse trabalho também se propôs a mostrar o comportamento diferenciado de genótipos de pimenteira-do-reino utilizando dois tipos de tutores no sistema de produção, o que o torna importante para estudos comparativos envolvendo a identificação de outros tipos de tutores para o cultivo dessa espécie.

Nesse trabalho, embora a produção de pimenta preta em tutor vivo seja inferior à obtida com o cultivo usando tutor morto (estacão) é viável seu uso, considerando os aspectos ecofisiológicos, de custo reduzido ao produtor na implantação do pimental, porém existe a necessidade de estudos complementares para melhor definição de um sistema de produção.

O sistema de produção de pimenta-do-reino em tutor vivo é uma realidade e vem sendo utilizada por alguns pipericultores, considerando o elevado valor das estacas, além da sustentabilidade ambiental, por não ser necessário o corte de árvores fornecedoras de madeira de lei, promover a redução da temperatura do solo e incorporar nutrientes pela utilização dos restos da gliricídia utilizada como cobertura morta, resultante do processo de poda. No entanto, há necessidade de estudar as interações entre a pimenteira-do-reino e gliricídia, com relação à necessidade de nutrientes e água para que se possa compor um sistema de cultivo mais efetivo.

\section{CONCLUSÕES}


Nas condições edafoclimáticas do município de Igarapé Açu, as cultivares de pimenteira-do-reino têm maior produção em tutor morto, com exceção da cv. Embrapa que se desenvolve bem em tutor vivo (gliricídia).

A cultivar Uthirankotta se destaca no cultivo em tutor vivo comparando-se aos demais materiais.

O cultivo da pimenteira-do-reino em tutor vivo é viável e mantém uma estabilidade de produção ao longo do ciclo produtivo, além do custo reduzido para implantação.

\section{AGRADECIMENTOS}

Ao Banco da Amazônia (BASA) pelo financiamento do projeto (No. 3737).

\section{REFERÊNCIAS}

ALBUQUERQUE, F. C.; DUARTE, M. L. R. Comportamento de cultivares de pimenta-do-reino, em área de ocorrência de fusariose no Estado do Pará. Belém: EMBRAPA-CPATU, 1991. 40 p, (EMBRAPA-CPATU. Documentos, 59). ISSN 0101-2835.

ANDRADE, C. G. C.; SILVA, M. L.; SALLES, T. T. Fatores Impactantes no Valor Bruto da Produção de Pimenta-do-reino (Piper nigrum L.) no Pará. Floresta e Ambiente. [online]. v. 24, 2017, ISSN 1415-0980. http://dx.doi.org/10.1590/21798087.145615.

ASSIS, B. V. R.; MEIRA, F. O.; PINA, V. G. S. S.; ANDRADE, G. F.; COTRIM, B. A. et al. Efeito Inibitório do Extrato de Piper nigrum L. sobre a Corrosão do Aço Carbono em Meio Ácido. Revista Virtual de Química, n.7, v. 5, p. 1830-1840, 2015. ISSN: $1984-6835$.

CONCEIÇÃO, H. E. O. da; FRAZÃO, D. A. C.; VIÉGAS, I. de J. M.; POLTRONIERI, M. C.; SARMANHO, F. R. S. et al. Comportamento de cultivares de pimenteirasdo-reino em sistema de cultivo com uso de tutor vivo. 5p., 2003.

https://www.embrapa.br/en/busca-de-publicacoes/-

/publicacao/408112/comportamento-de-cultivares-de-pimenteiras-do-reino-emsistema-de-cultivo-com-uso-de-tutor-vivo.

FILGUEIRAS, G. C.; HOMMA, A. K. O.; SANTOS, M. A. S. Conjuntura do mercado da pimenta-do-reino no Brasil e no mundo. [Online]. Belém. Embrapa; 2014. http://ainfo.cnptia.embrapa.br/digital/bitstream/item/43563/1/Mercado.pdf.

FRANZINI, V. I.; SILVA, A. R. B.; BOTELHO, S. M. Área de plantio, Calagem e Adubação. In: Boas práticas agrícolas para aumento da produtividade e qualidade da pimenta-do-reino no Estado do Pará. Brasília, DF: Embrapa, v. 52 p. 2014. ISBN 978-85-7035-305-4.

FREIRE, R. R. Diagnóstico da produção de mudas em viveiros registrados e propagação vegetativa da pimenteira-do-reino (Piper nigrum L.) no norte do Espírito Santo. Dissertação de mestrado. Universidade Federal do Espírito Santo. São Mateus- Espírito Santo, p. 90, 2013. 
IBGE - Instituto Brasileiro de Geografia e Estatística. Levantamento Sistemático da produção Agrícola (LSPA): Pesquisa Mensal de Previsão e Acompanhamento das Safras Agrícolas no Ano Civil. Fundação Instituto Brasileiro de Geografia e Estatística. Rio de Janeiro, v. 30 n.1 p.1-81, janeiro 2017. ISSN 0103-443X. ftp://ftp.ibge.gov.br/Producao_Agricola/Levantamento_Sistematico_da_Producao_Ag ricola_\%5Bmensal\%5D/Fasciculo/lspa_201701.pdf.

INMET - Instituto Nacional de Meteorologia. Precipitação total anual. 2014, 2015 e $2016 . \quad$ Disponível em: <http://www.inmet.gov.br/portal/index.php?r=clima/page\&page=desvioChuvaAnual>. Acesso em: 4 fev.2017.

ISHIZUKA, Y.; KATO, A. K.; CONCEIÇÃO, H. E. O. da; DUARTE, M. de L. R. Sistema de cultivo sombreado. In: DUARTE, M. de L. R. Cultivo da pimenteira-doreino na Região Norte. Belém, PA: Embrapa Amazônia Oriental. Sistemas de produção, v. 1, p. 83-89, 2004. ISSN 1983-0513.

KATO, A. K., UCHIDA, M., MENEZES, A. J. E. A., OGATA, T., ALBUQUERQUE, F. C., HAMADA, M., DUARTE, M. L. R. Utilização de tutores vivos na cultura da pimenta-do-reino. In: Seminário Internacional sobre Pimenta-do-reino e cupuaçu, v. 1, 1996. Belém, PA. Anais. Belém: Embrapa Amazônia Oriental/JICA, Embrapa Amazônia Oriental. Documentos, v. 89, p.435-440, 1997. ISSN 0101-2835.

MENEZES, A. J. E. A. de. Tutor vivo com gliricídia. In: Boas práticas agrícolas para aumento da produtividade e qualidade da pimenta-do-reino no Estado do Pará. Brasília, DF: Embrapa, v. 52 p. 2014. ISBN 978-85-7035-305-4.

MENEZES, A. J. E. A. de; HOMMA, A. K. O.; ISHIZUKA, Y.; KODAMA, N. R.; KODAMA, E. E. Gliricídia como tutor vivo para pimenteira-do-reino. Embrapa Amazônia Oriental. Série documentos 393. Belém, PA: Embrapa Amazônia Oriental, 31p., 2013. ISSN 1983-0513.

MENEZES, A. J. E. A. de; HOMMA, A. K. O.; ISHIZUKA, Y.; KODAMA, N. R.; KODAMA, E. E. Cultivo da pimenteira-do-reino (Piper nigrum L.) com tutor vivo de gliricídia (Gliricidia sepium L.) para produção no Estado do Pará. Revista de Agropecuária da Embrapa Amazônia Oriental - Ano II - no 3, 2016. https://www.infoteca.cnptia.embrapa.br/infoteca/bitstream/doc/1060835/1/Agrofocop2 2.pdf.

NELSON, S.; EGER, K. Black pepper (Piper nigrum). In C. R. Elevitch (Ed.) Farm and Forestry Production and Marketing Profile for Black Pepper (Piper nigrum). p. 14. Holualoua: Permanent Agriculture Resources (PAR), 2011.

OliveIRA, A. P.; ALVES, E. U.; SILVA, J. A.; ALVES, A. U.; OlIVEIRA, A. N. P.; LEONARDO, F. A. P.; MOURA, M. F.; CRUZ, I. S. Produtividade da pimenta-doreino em função de doses de esterco bovino. Horticultura Brasileira, 25: 408-410. 2007. ISSN 0102-0536. 
PEREIRA LOURINHO, M.; COSTA, C. A. S.; SOUZA, L. C.; SOUZA, L. C.; OLIVEIRA, C. F. Conjuntura da pimenta-do-reino no mercado nacional e na região Norte do Brasil.Enciclopédia Biosfera, v.10, n.18, p. 1016-1031, 2014. http://www.conhecer.org.br/enciclop/2014a/AGRARIAS/conjuntura\%20da\%20piment a.pdf.

POLTRONIERI, M. C. LEMOS, O. F. Cultivares. In: Boas práticas agrícolas para aumento da produtividade e qualidade da pimenta-do-reino no Estado do Pará. Brasília, DF: Embrapa, v. 52 p. 2014. ISBN 978-85-7035-305-4.

RODRIGUES, J. E. L. F.; LUCCHESI, A. A.; VIANA, F. M. P.; ALBUQUERQUE, F. C.; DUARTE, M. L. R.; MEDRADO, M. J. S. Estudo comparativo da incidência de antracnose (Colletotrichum gloeosporioides) em cultivares de pimenta-do-reino (Piper nigrum L.), recém introduzidos em porto velho, Rondônia. In: Anais da E. S. A. $\begin{array}{llll}\text { Luiz de Queiroz. V. XLIV-1987, pp.687-693. } & 1987 .\end{array}$ http://www.scielo.br/pdf/aesalq/v44n1/36.pdf

VELOSO, C. A. C.; CARVALHO, E. J. M.; MALAVOLTA, E.; MURAOKA, T. Resposta de cultivares de pimenta-do-reino aos nutrientes NPK em um Latossolo Amarelo da Amazônia Oriental. Scientia Agricola. [online]. v.57, n.2, pp.343-347. 2000. ISSN 0103-9016. 